\title{
Enhanced Plasticity of Retinothalamic Projections in an Ephrin-A2/ A5 Double Mutant
}

\author{
Alvin W. Lyckman, ${ }^{1}$ Sonal Jhaveri, ${ }^{1}$ David A. Feldheim, ${ }^{2}$ Pierre Vanderhaeghen, ${ }^{2,3}$ John G. Flanagan, ${ }^{2}$ and \\ Mriganka Sur ${ }^{1}$ \\ 1Department of Brain and Cognitive Science, Massachusetts Institute of Technology, Cambridge, Massachusetts 02139, \\ 2Department of Cell Biology and Program in Neuroscience, Harvard Medical School, Boston, Massachusetts 02115, and \\ 3/nstitute of Interdisciplinary Research, University of Brussels, B-1070 Brussels, Belgium
}

\begin{abstract}
Ascending sensory information reaches primary sensory cortical areas via thalamic relay neurons that are organized into modality-specific compartments or nuclei. Although the sensory relay nuclei of the thalamus show consistent modalityspecific segregation of afferents, we now show in a wild-type mouse strain that the visual pathway can be surgically "rewired" so as to induce permanent retinal innervation of auditory thalamic cell groups. Applying the same rewiring paradigm to a transgenic mouse lacking the EphA receptor family ligands ephrin-A2 and ephrin-A5 results in more extensive rewiring than in the wild-type strain. We also show for the first time that ephrin-A2 and ephrin-A5 define a distinct border between visual and auditory thalamus. In the absence of this ephrin-A2/A5
\end{abstract}

Visual, auditory, and somatosensory information ascend the CNS along discrete channels and are ultimately relayed to specific primary cortical areas via anatomically discrete, modality-specific compartments of thalamic relay neurons. The developmental mechanisms responsible for establishing these subcortical modality-specific thalamic relay pathways are poorly understood. It is possible, for instance, that each modality bears a separate set of molecular markers that are complementary between ascending afferents and their appropriate target relay neurons. Although putative recognition molecules, such as the cadherins (Suzuki et al., 1997; Inoue et al., 1998; Shapiro and Colman, 1999), and guidance molecules, such as the ephrins and their Eph receptor tyrosine kinases (Feldheim et al., 1998; Donoghue and Rakic, 1999a; Mellitzer et al., 1999; Xu et al., 1999; Vanderhaeghen et al., 2000), have been shown to be differentially expressed in different neural compartments of the CNS, there are no known examples of exclusive, modality-specific markers.

Innervation of sensory thalamic compartments by ascending sensory inputs is normally highly stereotyped, but it can be profoundly altered by genetic or surgical manipulations occurring early in development. In altricial species such as hamster (Schneider, 1973; Kalil and Schneider, 1975; Frost, 1981; Bhide and Frost, 1999) and ferret (Sur et al., 1988; Roe et al., 1990), early postnatal deafferentation of the auditory thalamic cell group, the

Received June 6, 2001; revised July 20, 2001; accepted July 20, 2001.

This work was supported by National Institutes of Health Grant EY 11512 and a grant from the March of Dimes. We thank Christine Waite for technical assistance and Dr. Larry I. Benowitz for his support during the initial stages of this work.

Correspondence should be addressed to Dr. Alvin W. Lyckman, Center for Learning and Memory, Massachusetts Institute of Technology, Building E25, Room 235, 45 Carleton Street, Cambridge, MA 02139. E-mail: lyckman@mit.edu.

Copyright (C) 2001 Society for Neuroscience $0270-6474 / 01 / 217684-07 \$ 15.00 / 0$ border and after rewiring surgery, retinal afferents are better able to invade and innervate the deafferented auditory thalamus. These data suggest that signals that induce retinal axons to innervate the denervated auditory thalamus may compete with barriers, such as the ephrins, that serve to contain them within the normal target. The present findings thus show that the targeting of retinothalamic projections can be surgically manipulated in the mouse and that such plasticity can be controlled by proteins known to regulate topographic mapping.

Key words: medial geniculate body; medial geniculate nucleus; inferior colliculus; cross-modal; rewiring; compartmentalization; ephrins; Eph receptors; target specificity

medial geniculate body (MGB), results in its cross-modal innervation, or "rewiring," by retina. Deafferentation of the somatosensory cell group, the ventrobasal complex, also results in its permanent innervation by retina (Frost, 1981). Conversely, the visual thalamic cell group, the lateral geniculate body (LGB), receives inputs from auditory afferents in a species that has early congenital degeneration of retina (Bronchti et al., 1989; Doron and Wollberg, 1994).

To begin probing developmental mechanisms that regulate modality-specific compartmentalization, we successfully applied a surgical rewiring paradigm to the mouse. Because compartmentalization could be mediated by axonal repulsion, we focused attention on thalamic regions in which retinal axons bearing Eph receptors (Cheng et al., 1995) might encounter high levels of ephrin proteins (Cheng et al., 1995; Drescher et al., 1995), i.e., regions from which retinal axons should be repelled (Drescher et al., 1997; Flanagan and Vanderhaeghen, 1998). We found such a pattern of expression along the optic tract, at the border between the LGB and MGB. At this border, ephrin-A is heavily expressed within the MGB but not within the LGB. Examining innervation of the MGB by retina in an ephrin-A2/A5 double knock-out mouse (Feldheim et al., 2000), we found that retinal fibers do not show novel innervation of the MGB in non-operated mutants but that the retino-MGB innervation after surgical rewiring is greater in the double knock-out than in the wild type. These data suggest that innervation of novel targets by retinal afferents is significantly influenced by the balance of locally expressed attractant and repellant factors, including the ephrins.

Parts of this work have been published previously in abstract form (Lyckman et al., 1999). 


\section{MATERIALS AND METHODS}

Animals. Axonal tracing and rewiring surgeries were performed on two mouse strains: (1) wild-type 129/SvEv mice (serving as controls) obtained from Taconic Farms (Germantown, NY) that were bred and maintained in our in-house colony [Massachusetts Institute of Technology (MIT), Division of Comparative Medicine]; and (2) ephrin-A2/A5 double knock-out mice (Feldheim et al., 2000) that were generated at Harvard Medical School. The double knock-outs were obtained by crossing a homozygous ephrin-A2 knock-out in a pure 129/SvEv background (Feldheim et al., 1998, 2000) with a homozygous ephrin-A5 knock-out in a mixed Swiss-Webster/C57BL/6 background (Frisén et al., 1998). Live animal procedures were approved by the Committee on Animal Care at MIT and conformed to National Institutes of Health guidelines.

Auditory axon tracing. Postnatal day 0 (P0) mice were killed with sodium pentobarbital $(100 \mathrm{mg} / \mathrm{kg})$ and transcardially perfused with saline and 4\% paraformaldehyde in PBS. Minute crystals of DiI (Molecular Probes, Eugene, OR) were placed at the confluence of the brachium of the inferior colliculus (BIC) and the lateral lemniscus (LL) in fixed P0 mouse brains. Brains were placed in fixative at $25^{\circ} \mathrm{C}$ in the dark for $6-8$ weeks. Brains were photographed with bright-field optics in whole mount and then sectioned in the sagittal plane at $150 \mu \mathrm{m}$ with a vibratome. Sections were mounted in Vectashield (Vector Laboratories, Burlingame, CA) and digitally imaged under epifluorescence.

Rewiring surgery. Neonatal (P0 or P1) mice were deeply anesthetized by hypothermia. A longitudinal incision in the scalp was made directly over the midbrain. The inferior colliculus (IC) and the superficial layers of the superior colliculus (SC) were ablated by microcautery. The BIC was severed by making a short, transverse, midcollicular incision with a microknife. After lesioning, the scalp was sutured with 7-0 silk, and the neonate was gently rewarmed before being returned to the nest.

Retinal axon tracing. Retinal axons were traced using $1 \mu l$ intraocular injections of $1 \%$ cholera toxin B-subunit (CTB), followed by tissue processing and immunohistochemistry as described previously (Angelucci et al., 1996; Ling et al., 1998) with the following exceptions. Mice were anesthetized with Avertin (Aldrich, Milwaukee, WI). Peroxidase was revealed with either VIP (Vector Laboratories) according to the instructions of the manufacturer or $0.01 \%$ diaminobenzidine (Sigma, St. Louis, MO) and $0.01 \% \mathrm{H}_{2} \mathrm{O}_{2}$ in PBS. A set of sections was counterstained with thionin. For whole-mount staining of the optic tract in neonates, mouse pups were deeply anesthetized by hypothermia and intraocularly injected with $1 \mu \mathrm{l}$ of $5 \%$ wheat germ agglutinin-coupled horseradish peroxidase in PBS. One day after injection, pups were killed and perfused as above. Brains were dissected out, and cerebral hemispheres were removed. Peroxidase was revealed using tetramethylbenzidine (Angelucci et al., 1996).

Ephrin-A and EphA expression. Expression patterns of ephrin-A proteins and EphA receptor tyrosine kinases were stained using alkaline phosphatase (AP)-coupled affinity probes (Cheng et al., 1995; Feldheim et al., 1998). To identify the subtypes of ephrin-A proteins revealed using the affinity probes, we processed mouse brain sections for in situ hybridization using antisense riboprobes for ephrin-A2 and ephrin-A5 and revealed with digoxigenin immunohistochemistry (Cheng et al., 1995; Feldheim et al., 1998; Vanderhaeghen et al., 2000).

Quantitative analyses of cross-modal rewiring. The extent of rewiring in the wild-type and double knock-out groups was quantitated by digital image analysis. First, digital images from complete series of coronal sections through the MGB of individual cases stained for CTB were cropped to delete pixels not within the histological boundaries of MGB; these cropped images were then normalized to 256 levels of gray. The normalized images of MGB were then binarized so that pixel values of 0-128 (those pixels considered background) were set to white, and those with pixel values $>128$ (i.e., those representing CTB signal) were set to black. Two statistics were then determined: volume of rewiring and extent of rewiring. For volume measurements, the black pixels from all sections from an individual case were summed; such pixel sums represent the volume of innervation of MGB by retina in individual cases. These pixel sums were then converted to cubic micrometers to yield estimates of actual volumes of retino-MGB innervation in individual cases. For extent measurements, we asked how far were the deepest retinal terminals in MGB from the lateral border of the MGB. To do this, we determined the lateral border of the MGB and then found the terminals most distant from it along lines perpendicular to line segments that were tangential to this lateral border. The greatest such extent was determined for each individual case.
Digital microscopy and image analyses. Bright-field images were captured using a Sony (Tokyo, Japan) MDK-5000 color digital camera attached to a Leitz (Wetzlar, Germany) Diaplan or to a Wild Makroskop. Fluorescent images were captured using a Kodak (Eastman Kodak, Rochester, NY) digital camera attached to a Nikon (Tokyo, Japan) microscope. Images were processed using Photoshop (Adobe Systems, San Jose, CA) and montaged with Canvas (Deneba Systems Inc., Miami, FL). NIH Image was used for densitometric analyses of ephrin gradients in MGB and for morphometry.

\section{RESULTS}

\section{Retinal innervation of auditory thalamus in wild-type mouse: cross-modal rewiring}

The ascending, subcortical visual pathway consists of axons of retinal ganglion cells that course along the optic tract to the SC (Fig. 1a). In dorsal thalamus, these axons send collateral branches into dorsal and ventral nuclei of the LGB (LGd and LGv, respectively). Relay neurons in the LGd in turn project to the primary visual cortex (Fig. 1a, V1). Ascending auditory information reaches the auditory relay center of the thalamus, the MGB, via axons running in the BIC from neurons in the IC (Fig. 1a). We suspected rewiring lesions need be done early in mice, but there were no indications in the literature that the auditory pathway was in place by $\mathrm{P} 0$ in mice. Axon tracing using DiI in fixed $\mathrm{P} 0$ mouse brains revealed the presence of an abundance of axons coursing through the lateral lemniscus to the IC and along the BIC between the IC and the MGB (Fig. 1b). Although it is well documented that retinal axons robustly innervate LGd and LGv in neonatal mouse (Godement et al., 1984), we also do not find retinal axon terminals in MGB in unlesioned mice of either the control 129/SvEv strain (Fig. 1c) (see Fig. 3a) or in the ephrinA2/A5 double knock-out strain (Fig. 1d) (see Fig. 3d) at any of the postnatal time points we examined (P1, P5, P14, and adult) using the highly sensitive CTB technique (Angelucci et al., 1996; Ling et al., 1998).

In initial rewiring surgeries with $129 / \mathrm{SvEv}$ wild-type mice, the MGB was deprived of auditory afferents by unilateral ablation of the IC and section of the BIC on P0 (Fig. 1e). As per previous rewiring studies in hamster and ferret, the SC was also lesioned to reduce the amount of normal target area available to growing retinal axons. Operated animals were allowed to survive for at least 12 d. Lesions were confirmed histologically (Fig. 1f). As revealed by CTB tracing, the MGB ipsilateral to the lesion was clearly innervated by retinal axons (Fig. $1 g$ ). Retinal axon terminals were typically clustered in MGB in proximity to the optic tract, i.e., in the lateral portion of the MGB, but could be found upward of $220 \mu \mathrm{m}$ from the lateral edge of MGB. These findings (additional examples of which are given in Fig. 3b,c) demonstrate that the pattern of retinothalamic targeting in mice can be altered by a surgical rewiring paradigm that had been thought to require very immature, altricial species (Kalil and Schneider, 1975; Sur et al., 1988).

To better understand the role of normal target availability on the potential for inducing retinal axons to innervate the denervated MGB, rewiring lesions $(n=2)$ were done in which the IC was ablated (Fig. 1h) while the SC was essentially spared (Fig. 1i). Retinal axon tracing with CTB after these SC-sparing lesions revealed considerable retinal innervation of MGB (Fig. 1j). These findings demonstrate that cross-modal rewiring of MGB by retina can occur despite an intact retinal target field. This suggests that retinal axons innervate novel targets (such as MGB) not because of a lack of normal target space (Frost, 1981; Sabel and Schneider, 

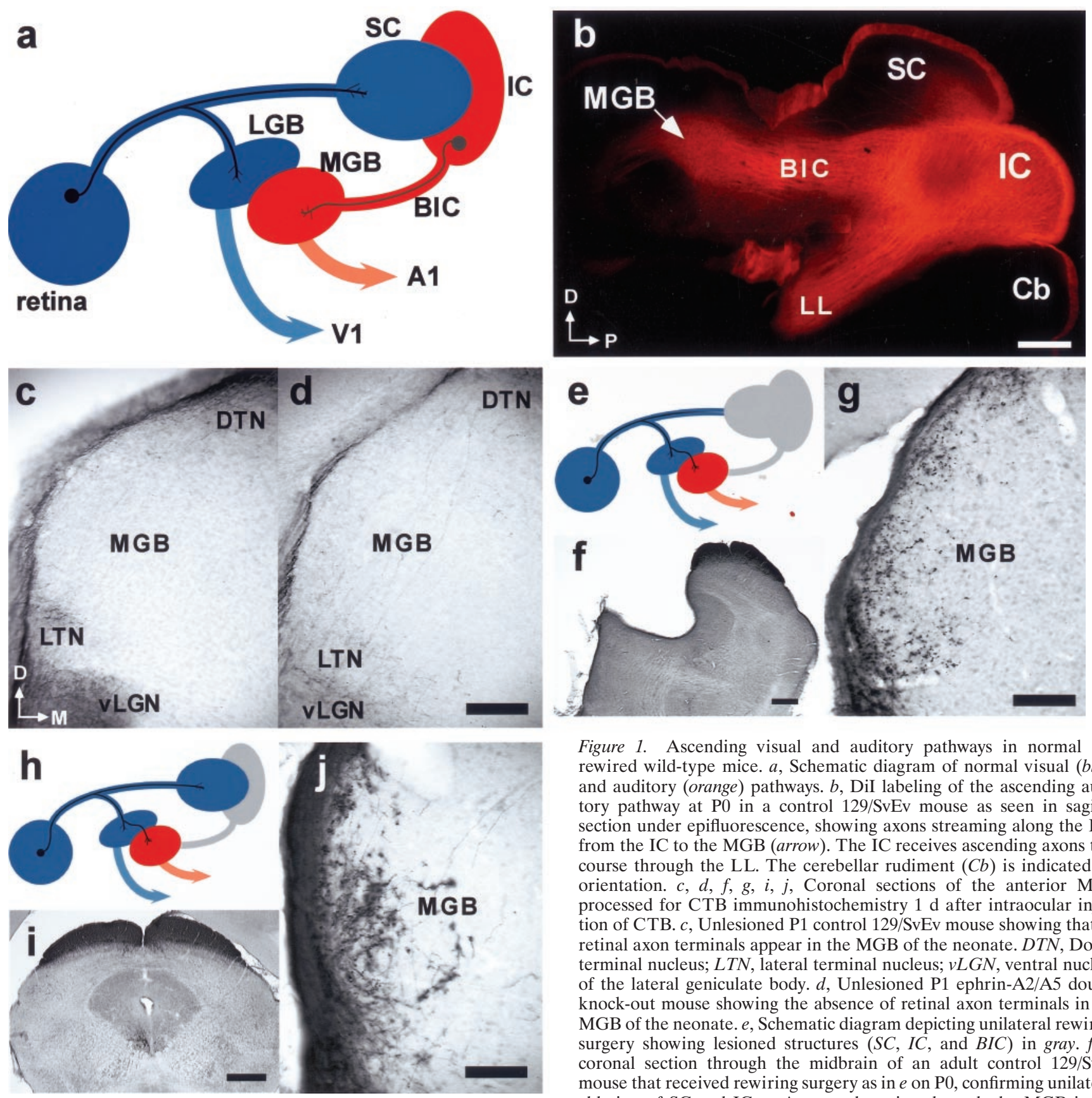

Figure 1. Ascending visual and auditory pathways in normal and rewired wild-type mice. $a$, Schematic diagram of normal visual (blue) and auditory (orange) pathways. $b$, DiI labeling of the ascending auditory pathway at P0 in a control $129 / \mathrm{SvEv}$ mouse as seen in sagittal section under epifluorescence, showing axons streaming along the BIC from the IC to the MGB (arrow). The IC receives ascending axons that course through the LL. The cerebellar rudiment $(\mathrm{Cb})$ is indicated for orientation. $c, d, f, g, i, j$, Coronal sections of the anterior MGB processed for CTB immunohistochemistry $1 \mathrm{~d}$ after intraocular injection of CTB. $c$, Unlesioned P1 control $129 / \mathrm{SvEv}$ mouse showing that no retinal axon terminals appear in the MGB of the neonate. DTN, Dorsal terminal nucleus; $L T N$, lateral terminal nucleus; $v L G N$, ventral nucleus of the lateral geniculate body. $d$, Unlesioned P1 ephrin-A2/A5 double knock-out mouse showing the absence of retinal axon terminals in the MGB of the neonate. $e$, Schematic diagram depicting unilateral rewiring surgery showing lesioned structures $(S C, I C$, and $B I C)$ in gray. $f, \mathrm{~A}$ coronal section through the midbrain of an adult control $129 / \mathrm{SvEv}$ mouse that received rewiring surgery as in $e$ on $\mathrm{P} 0$, confirming unilateral ablation of SC and IC. $g$, A coronal section through the MGB in the same mouse as in $f$. Punctate staining within MGB indicates the presence of retinal axon terminals. $h$, Schematic diagram depicting rewiring surgery in which the IC and BIC were lesioned (shown in gray), but the SC was spared. $i$, Coronal section at the midcollicular level from an adult control $129 / \mathrm{SvEv}$ mouse that was lesioned as in $h$ on P0, confirming the sparing of SC (lesioned side to the left). $j$, The MGB in the same mouse as in $h$ showing retinal innervation of the MGB induced by lesioning the IC. Scale bars: $b, d, 100 \mu \mathrm{m} ; f, g, i, j, 150 \mu \mathrm{m}$. $D$, Dorsal; $M$, medial; $P$, posterior.

1988) but rather as a result of changes in signals emanating specifically from adjacent, abnormally denervated targets.

\section{Ephrin-A expression in visual and auditory thalamus}

The ability of retinal axons to innervate a novel, denervated target suggests that denervation might alter signals that serve to attract growing afferents and/or signals that serve to repel growing afferents. As a prominent example of the latter, the ephrin-A ligands had been shown to regulate topographic patterning (Drescher et al., 1995; Nakamoto et al., 1996; Monschau et al., 1997; Feldheim et al., 1998, 2000; Frisén et al., 1998) and target containment (Frisén et al., 1998) by repulsion of retinal afferents bearing EphA receptors. We therefore asked whether the pattern of expression of ephrin-A ligands was related to containment of retinal afferents to the LGB. Expression of EphA receptors and ephrin-A ligands was examined in the thalamus in whole mounts and vibratome slices of P0-P14 mouse brain using alkaline phosphatase-coupled affinity probes in situ (Cheng et al., 1995; Feldheim et al., 1998). As revealed by ephrin-A5 affinity-probe staining, expression of EphA receptors (Fig. $2 a^{\prime}$ ) is tightly colocalized with the optic tract (Fig. $2 a$ ). The MGB, BIC, and IC are free of labeled retinal axons (Fig. $2 a$ ) and show virtually no detectable expression of EphA receptors with ephrin-A5 affinity-probe staining (Fig. $2 a^{\prime}$ ). 


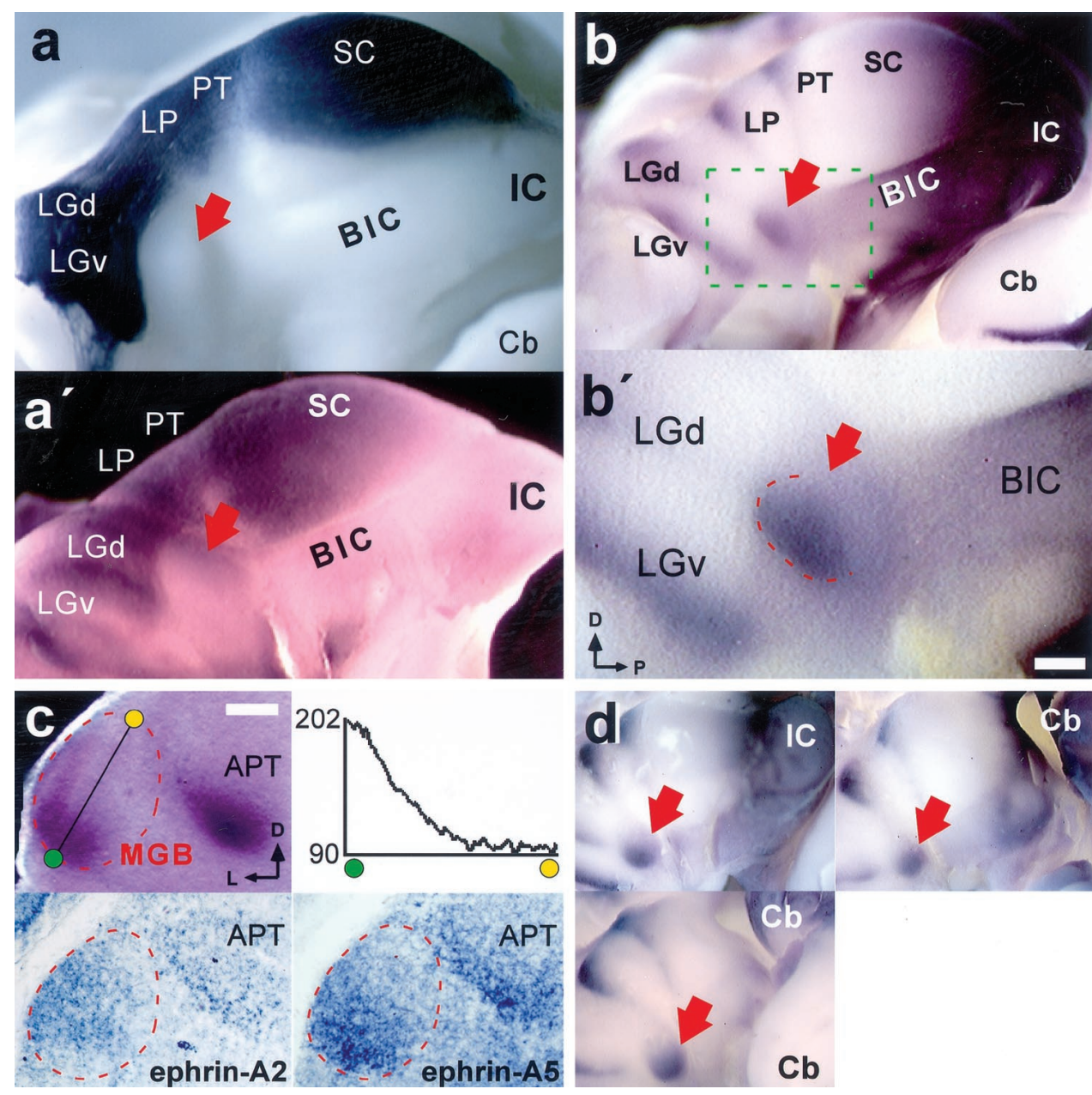

Figure 2. Neonatal expression of EphA and ephrin-A in visual and auditory thalamus. $a, a^{\prime}, b, b^{\prime}, d$, Lateral views of the diencephalon and midbrain in whole mount, oriented dorsal $u p$ and posterior to the right. The cortex was removed before staining reactions. Red arrows point to the MGB. The cerebellar rudiment $(\mathrm{Cb})$ is noted for orientation. $a$, Optic tract and associated nuclei ( $L G d$ and $L G v$ ); the lateral posterior nucleus $(L P)$, the pretectal nuclei $(P T)$, and the SC as labeled by anterograde tracing with wheat germ agglutinin-coupled-HRP. $a^{\prime}$, EphA receptor expression at $\mathrm{P} 0$ as revealed by ephrinA5-AP affinity-probe staining. Note that this staining tightly matches that seen in $a$. $b$, Ephrin-A ligand expression at $\mathrm{P} 0$ as revealed by EphA3-AP affinity-probe staining. Note the moderately dense staining in the nuclei along the optic tract, in the MGB, and along the BIC and intense staining of the IC. $b^{\prime}$, Enlargement of boxed region in $b$ showing the $\mathrm{LGB}, \mathrm{MGB}$, and BIC. Graded expression of ephrin-A in MGB is evident, as well as a distinct border of expression in the MGB at the boundary between the posterior margin of LGB and the anterior margin of the MGB (demarcated by the dashed red curve). $c$, Staining for ephrin-A protein and mRNA in coronal sections through the anterior MGB (encircled by red dashed line) at P0. Top left, Staining with EphA3-AP affinity probe in a coronal vibratome section. Top right, The pixel intensity profile of EphA3-AP affinityprobe staining through the MGB along the black line from the green circle to the yellow circle. $y$-Axis indicates gray scale pixel values. Bottom, In situ hybridization for ephrin-A2 (left) and ephrin-A5 (right) in

cryostat sections. The position of the anterior pretectal nucleus $(A P T)$ is given for orientation. Lateral is to the left. $d$, EphA3-AP affinity-probe staining in laterally viewed whole mounts after rewiring lesions on P0. Top, Control side (left) and lesion side (right) $3 \mathrm{~d}$ after unilateral ablation of SC and IC. Bottom, Left side after bilateral ablation of IC and SC $3 \mathrm{~d}$ after lesioning. $D$, Dorsal; $L$, lateral; $P$, posterior. Scale bars: $a^{\prime}, 200 \mu \mathrm{m} ; b^{\prime}, c, d, 50 \mu \mathrm{m}$.

As revealed by EphA3 affinity-probe staining, ephrin-A proteins are expressed in several thalamic and midbrain nuclei as a series of discrete, localized gradients in LGv, LGd, the lateral posterior nucleus, the pretectal region, and the SC (Fig. 2b). At the ventroposterior border of LGB, the gradient of ephrin-A ligands fades at its border with MGB, at the point in MGB at which its ephrin-A gradient is strongest (Fig. $2 b^{\prime}$ ). Thus, the border between LGB (and the overlaying optic tract) and the MGB (Fig. 2b', dashed curve) is coincident with the high end of a molecular gradient of ephrin-A ligands in MGB. Staining with EphA3 affinity probe for ephrin-A in coronal vibratome sections reveals that the ephrin-A gradient extends mediolaterally and dorsoventrally into the MGB (Fig. $2 c$, top). In situ hybridization staining reveals expression of ephrin-A2 (Fig. 2c, bottom left) and ephrin-A5 (Fig. $2 c$, bottom right) mRNAs in MGB. Ephrin-A2 mRNA is expressed in a slight gradient that is most dense at the lateral edge of MGB, diminishing in the medial direction, that may contribute to the lateral-most affinity-probe staining. Ephrin-A5 mRNA is expressed in a steep gradient, running $\sim 30^{\circ}$ to the ventrodorsal axis, that also closely matches the affinityprobe staining. The densest retinal innervation of MGB in the rewiring cases (Figs. 1g,j, $3 b, c, e, f$ ) is frequently in the region of the MGB where ephrin-A proteins (Fig. 2c, top left) and mRNAs (Fig. 2c, bottom) are at their highest levels of expression.
Ephrin-A proteins appear to be expressed along the BIC (Fig. $\left.2 b, b^{\prime}\right)$, suggesting that they may be carried by fibers that connect the IC to the MGB. It was of interest to know whether expression of ephrin-A ligands in MGB derives from its innervation by IC. Mice that received rewiring surgeries on $\mathrm{P} 0$ were prepared for whole mount EphA3 affinity-probe histochemistry 2-4 d later. In one such case, ephrin-A ligands are detectable in MGB on both the unoperated side (Fig. 2d, top left) and operated side (Fig. 2d, top right) of a $\mathrm{P} 4$ brain. In another case, which received a bilateral rewiring surgery (ablation of left and right IC and SC), ephrin-A ligands are readily detected $3 \mathrm{~d}$ after lesioning (Fig. $2 d$, bottom). Thus, the MGB can express ephrin-A ligands despite ablation of ephrin-A-positive auditory afferents from IC.

\section{Cross-modal rewiring in an ephrin-A2/ephrin-A5 double knock-out mouse}

Deafferentation of the MGB does not reduce ephrin-A expression in the MGB [as revealed with the Eph3A-AP affinity-probe staining (Fig. 2d)] yet is necessary and sufficient (Fig. 1j) to induce retinal axons to innervate the MGB. However, it is possible that deafferentation in fact causes subtle changes in ephrin-A expression that are masked by the presence of multiple ephrin-A proteins. To test the hypothesis that ephrin-A proteins contribute to modality-specific containment of the retinothalamic projection, 

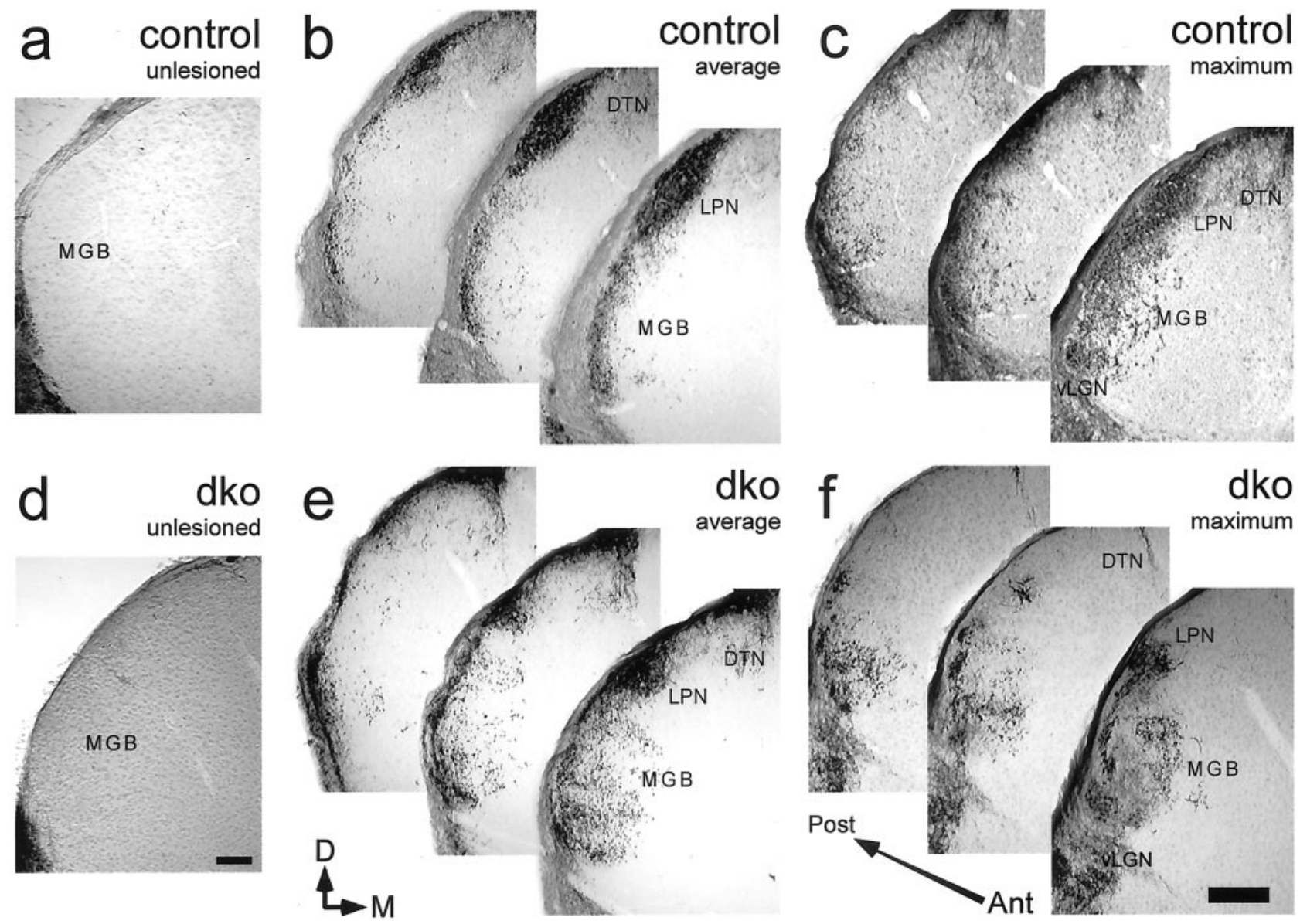

Figure 3. Retino-MGB innervation in control 129/SvEv versus ephrin-A2/A5 double knock-out mice, with and without rewiring surgery on $\mathrm{P} 0$, as revealed by anterograde tracing of retinal axon terminals using CTB immunohistochemistry. $a$, Unlesioned control 129/SvEv mouse. $b, c$, Serial sections from average $(b)$ and maximal $(c)$ cases of rewiring in the control 129/SvEv strain (based on the volume of innervation statistic; see Materials and Methods). $d$, Unlesioned ephrin-A2/A5 double knock-out mouse. $e, f$, Serial sections from average $(e)$ and maximal $(f)$ cases of rewiring in the ephrin-A2/A5 double knock-out strain (see Materials and Methods for details). In $b, c, e$, and $f$, the front-most section is anterior. The sections in $a$ and $d$ correspond to the middle sections in $b-d$ and $f$. In all panels, medial $(M)$ is to the right and dorsal $(D)$ is to the top. $d k o$, Double knock-out; $L P N$, lateral posterior nucleus; $D T N$, dorsal terminal nucleus; $v L G N$, ventral nucleus of the lateral geniculate body; Post, posterior; Ant, anterior. Scale bars: $d, f, 200 \mu \mathrm{m}$.

we reasoned that it would be necessary to apply the rewiring paradigm to a transgenic model deficient in both ephrin-A2 and ephrin-A5 (Feldheim et al., 2000). No EphA3 affinity-probe staining was detected in the thalamus or midbrain of this double knock-out strain (Feldheim et al., 2000), indicating that such staining in wild-type mice is primarily or exclusively attributable to expression of ephrin-A2 and ephrin-A5.

Anterograde staining of retinal ganglion cell axons in the control 129/SvEv strain and in the ephrin-A2/A5 double knockout strain shows that the retinothalamic projection is normally targeted to the thalamus in these double knock-out mice at early postnatal (Fig. 1c,d) and mature stages of development (Fig. $3 a, d)$. The surgical procedure that was effective in inducing crossmodal rewiring in the control $129 / \mathrm{SvEv}$ strain (Fig. 3b,c) was also very effective when applied to the ephrin-A2/A5 double knockout strain (Fig. $3 e, f$ ). To determine whether the combined absence of expression of ephrin-A2 and of ephrin-A5 had any impact on the pattern or extent of rewiring, we compared lesioned cases from both strains. Qualitatively, the pattern of retino-MGB innervation was similar in both strains; retinal terminals tended to be most abundant near the lateral edge of the MGB and in its anterior ventrolateral quadrant (Figs. 3b,c,e,f, $4 a, b)$. As noted previously in the hamster (Schneider, 1973; Frost,
1981; Sabel and Schneider, 1988), the lateral posterior nucleus also received an enhanced retinal projection after lesions of the superior colliculus (Fig. 3b,c,e,f).

For quantitative comparisons, we calculated two statistics: volume of rewiring (Fig. 4c), an absolute estimate of the amount of volume contributed by retinal axon terminal cytoplasm in MGB; and extent of rewiring (Fig. $4 d$ ), a measurement of the maximal penetration of retinal innervation into MGB from the lateral border of the MGB. Measurements were taken from control 129/SvEv mice and ephrin-A2/ephrin-A5 double knock-out mice that received unilateral rewiring surgery ( $n=6$, each group). The average volume of rewiring was $173 \%$ (2.7-fold) greater in the ephrin-A2/A5 double knock-out group $\left(3.50 \times 10^{6} \mu \mathrm{m}^{3} \pm 1.35 \times\right.$ $\left.10^{4} \mu \mathrm{m}^{3} \mathrm{SE}\right)$ than in the control 129/SvEv group $\left(1.28 \times 10^{6} \mu \mathrm{m}^{3}\right.$ $\left.\pm 5.10 \times 10^{3} \mu \mathrm{m}^{3} \mathrm{SE}\right)(p<0.028 ; t$ test; treating each case as a single datum) (Fig. 4c). Based on this volume statistic, average and maximal cases of rewiring are shown for the control 1 $29 / \mathrm{SvEv}$ strain in Figure 3, $b$ and $c$, respectively, and for the ephrin-A2/A5 double knock-out strain in Figure 3, $e$ and $f$, respectively. The average extent of rewiring was $27 \%$ (1.3-fold) greater in the ephrin-A2/A5 double knock-out group $(299 \pm 20$ $\mu \mathrm{m} \mathrm{SE})$ versus the control group $(236 \pm 19 \mu \mathrm{m} \mathrm{SE})(p<0.043$; $t$ test; treating each case as a single datum) (Fig. $4 d$ ). 

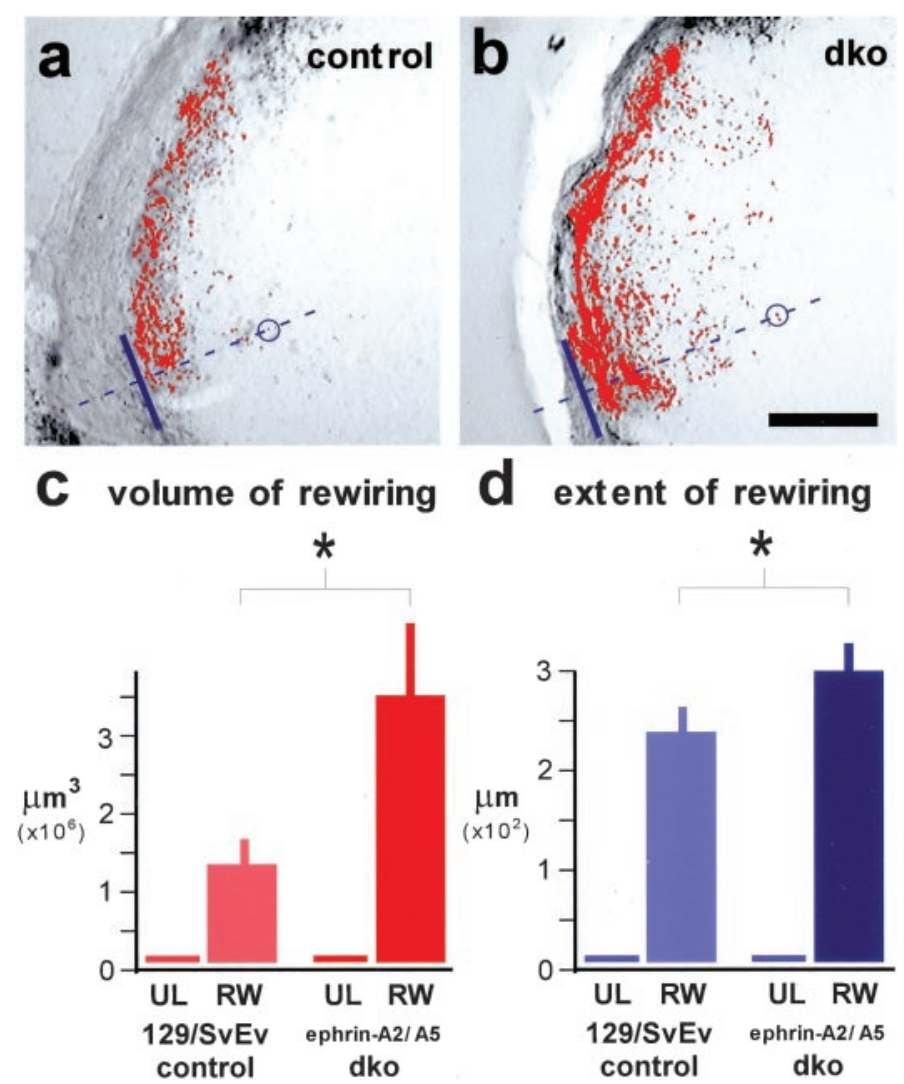

Figure 4. Quantitative analyses of rewiring in control 129/SvEv versus ephrin-A2/A5 double knock-out mice. $a, b$, Micrographs of sections (from average cases in Fig. 3) showing immunohistochemical detection of retino-MGB innervation ( $a$, control $129 / \mathrm{SvEv}$ case; $b$, ephrin-A2/A5 double knock-out case). The micrographs have been overlain to show the thresholded "black" pixels (in red); MGB border tangents (solid purple lines); perpendiculars to these tangents (dashed purple lines); and, the most distant retinal terminal along the perpendicular in each micrograph (centered within the purple circles). $c$, The average volume of rewiring in the control 129/SvEv strain (gray bars) versus the ephrin-A2/A5 double knock-out strain (black bars), calculated by summing the thresholded pixels in each individual case and then averaging these sums for six cases in each group. No retinal terminals were ever seen in the MGB of unlesioned mice of either strain. $d$, The average extent of rewiring in the control 129/SvEv strain (gray bars) versus the ephrin-A2/A5 double knock-out strain (black bars), calculated by finding the greatest distance from the lateral border of the MGB for each of six cases in each group. $U L$, Unlesioned; $R W$, rewired. Scale bar: $150 \mu \mathrm{m}$.

\section{DISCUSSION}

We found that two measures of retino-MGB innervation, the volume of innervation and the mediolateral extent of innervation, are significantly greater in rewired ephrin-A2/A5 double knockout mice than in control $129 / \mathrm{SvEv}$ mice. Some technical considerations are germane to these findings. The first is whether the $129 / \mathrm{SvEv}$ strain serves as a suitable control for the genetic background of the double knock-out strain. Our choice of the 129/ SvEv strain for control experiments was based on the fact that one of the founding single knock-outs, the ephrin-A2 $(-/-)$, is a pure $129 / \mathrm{SvEv}$ strain, whereas the other single knock-out, the ephrin-A5 (-/-), is at least partly derived from $129 / \mathrm{SvEv}$ stock. We are confident that there is significant genetic background shared between the 129/SvEv controls and the ephrin-A2/A5 double knock-outs. A additional practical consideration is that crosses between heterozygotes of the single knock-out strains would only generate double knock-outs (and controls) in 1/16 of the offspring, requiring extremely intensive, possibly prohibitive, breeding management to obtain suitable sample sizes. To see a maximal effect of ephrin-A proteins on rewiring, we chose to study the ephrin-A2/A5 double knock-out strain, a strain that lacks expression of the only two ephrin-A proteins known to be present in the dorsal thalamus of the wild type. By in situ hybridization, we show in fact that both are present in the MGB. Whether one of these contributes more to the observed effects cannot be judged from the present results, although ephrin-A5 mRNA is the more intensely labeled of the two in our material.

The basic molecular mechanisms that determine modalityspecific compartmentalization of thalamic inputs remain elusive. Nonetheless, considerable progress has been made in understanding the molecular control of topographic organization within a given projection (Drescher et al., 1997; Flanagan and Vanderhaeghen, 1998; Frisén et al., 1999). In the visual system, retinal fibers terminate with topographic order in the LGB, within which ephrin-A2 and ephrin-A5 are expressed as gradients that match the nasotemporal ordering of retinal fibers bearing EphA receptor tyrosine kinases (Cheng et al., 1995; Feldheim et al., 1998). In animals lacking either or both ephrin-A2 and ephrin-A5 proteins, retinogeniculate topography is specifically disrupted (Feldheim et al., 1998, 2000; Frisén et al., 1998). Ephrins have been shown to predict and/or mediate the topographic arrangements in other systems as well (Brownlee et al., 2000; Feng et al., 2000; Vanderhaeghen et al., 2000). A consistent theme in these functional analyses is that axons bearing high levels of Eph tyrosine receptor kinases are repelled from regions of high-level ephrin expression (Drescher et al., 1995; Nakamoto et al., 1996; Monschau et al., 1997; Feldheim et al., 1998; Frisén et al., 1998; Hornberger et al., 1999). Because specific ephrins show region-specific expression patterns in embryonic brain (Donoghue and Rakic, 1999a,b; Mackarehtschian et al., 1999), ephrins may not only have a role in ordering projections within target regions but might also serve to define different neural compartments. For instance, in mice lacking ephrin-A5 (Frisén et al., 1998), retinal axons form a transient aberrant projection to the IC, in which ephrin-A5 is normally intensely expressed in the wild type.

Although it is widely recognized that the retina robustly innervates its two main targets, the LGB and the SC, the repertoire of normal targets of the retinofugal projection is actually quite diverse (Ling et al., 1998). Thus, the retina innervates at least 25 distinct subcortical nuclei and cell groups. Conventional ideas of axonal targeting hold that axons are guided by specific mechanisms toward appropriate targets and that once contact between axons and appropriate target neurons is made, synaptogenesis ensues. Thus, recognition of appropriate target neurons for retinal ganglion cells has the complexities of the target diversity, laminar specificity (Godement et al., 1984; Upton et al., 1999), and topographic ordering (Bonhoeffer and Huf, 1982; Feldheim et al., 1998, 2000). Moreover, the present results document that, in mice, abnormal targeting is induced by denervation of a cell group adjacent to a normal target. This might suggest not only that growing retinal ganglion cell axons are unlikely to be predetermined to innervate fixed target cell types, such as relay neurons in LGd, but also that target cell type can be altered, i.e., that deafferented neurons in MGB can become retinorecipient. The present results demonstrate that retinal ganglion cell axons do not extend arbors and innervate foreign targets simply because of loss of their own target space; rewiring surgeries that spare the SC result in considerable retinal innervation of MGB. This finding 
points to the target as the major source for signals that regulate patterning of the retinothalamic projection.

An alternative to a rigid molecular prespecification of connections is a system wherein recognition depends on a complex system of competing determinants of recognition that nonetheless reliably results in highly stereotyped patterning. The present results are consistent with such a model. In the absence of ephrin-A ligands, rewiring surgery permits greater innervation of MGB by retina; whether this is attributable to greater numbers of retinal afferents invading MGB and/or more extensive arborization of a constant number of retinal afferents is not clear from the present data. That the retina fails to innervate MGB in non-operated animals even in the absence of ephrin-A ligands suggests that merely removing the ephrin-A barrier is alone insufficient to induce retinal axons to innervate the novel target; an attractant cue(s) must also be supplied by the denervation of MGB. The identities of such cues remain unknown at present. Importantly, our results suggest that attractant signals may compete with barriers such as the ephrins to regulate retinal projections to novel targets.

Finally, the presence of ephrin-A gradients in the MGB, as demonstrated in the present study, and in the auditory cortex (Vanderhaeghen et al., 2000) implicate this class of molecules in topographic mapping in the auditory pathway. Because retinal projections to the MGB are topographically patterned (Roe et al., 1991), as are projections from the MGB that specify a map of the retina in auditory cortex (Roe et al., 1990), the presence of ephrin-A gradients may also serve to pattern rewired visual projections. In the ephrin-A2/A5 double mutant, retinal fibers are dispersed more widely in their normal targets (Feldheim et al., 2000) and may be similarly widespread in the novel target, the MGB. Furthermore, these mice are likely to show considerable topographic disorder in the visual maps in rewired auditory cortex, as well as in their normal subcortical and cortical auditory maps (Vanderhaeghen et al., 2000).

\section{REFERENCES}

Angelucci A, Clasca F, Sur M (1996) Anterograde axonal tracing with the subunit B of cholera toxin: a highly sensitive immunohistochemical protocol for revealing fine axonal morphology in adult and neonatal brains. J Neurosci Methods 65:101-112.

Bhide PG, Frost DO (1999) Intrinsic determinants of retinal axon collateralization and arborization patterns. J Comp Neurol 411:119-129.

Bonhoeffer F, Huf J (1982) In vitro experiments on axon guidance demonstrating an anterior-posterior gradient on the tectum. EMBO J $1: 427-431$.

Bronchti G, Heil P, Scheich H, Wollberg Z (1989) Auditory pathway and auditory activation of primary visual targets in the blind mole rat (Spalax ehrenbergi). I. 2-deoxyglucose study of subcortical centers. J Comp Neurol 284:253-274.

Brownlee H, Gao PP, Frisén J, Dreyfus C, Zhou R, Black IB (2000) Multiple ephrins regulate hippocampal neurite outgrowth. J Comp Neurol 425:315-322.

Cheng HJ, Nakamoto M, Bergemann AD, Flanagan JG (1995) Complementary gradients in expression and binding of ELF-1 and Mek4 in development of the topographic retinotectal projection map. Cell 82:371-381.

Donoghue MJ, Rakic P (1999a) Molecular gradients and compartments in the embryonic primate cerebral cortex. Cereb Cortex 9:586-600.

Donoghue MJ, Rakic P (1999b) Molecular evidence for the early specification of presumptive functional domains in the embryonic primate cerebral cortex. J Neurosci 19:5967-5979.

Doron N, Wollberg Z (1994) Cross-modal neuroplasticity in the blind mole rat Spalax ehrenbergi: a WGA-HRP tracing study. NeuroReport 5:2697-2701.

Drescher U, Kremoser C, Handwerker C, Loschinger J, Noda M, Bonhoeffer F (1995) In vitro guidance of retinal ganglion cell axons by RAGS, a $25 \mathrm{kDa}$ tectal protein related to ligands for Eph receptor tyrosine kinases. Cell 82:359-370.

Drescher U, Bonhoeffer F, Muller BK (1997) The Eph family in retinal axon guidance. Curr Opin Neurobiol 7:75-80.
Feldheim DA, Vanderhaeghen P, Hansen MJ, Frisén J, Lu Q, Barbacid M, Flanagan JG (1998) Topographic guidance labels in a sensory projection to the forebrain. Neuron 21:1303-1313.

Feldheim DA, Kim YI, Bergemann AD, Frisén J, Barbacid M, Flanagan JG (2000) Genetic analysis of ephrin-A2 and ephrin-A5 shows their requirement in multiple aspects of retinocollicular mapping. Neuron 25:563-574.

Feng G, Laskowski MB, Feldheim DA, Wang H, Lewis R, Frisen J, Flanagan JG, Sanes JR (2000) Roles for ephrins in positionally selective synaptogenesis between motor neurons and muscle fibers. Neuron 25:295-306.

Flanagan JG, Vanderhaeghen P (1998) The ephrins and Eph receptors in neural development. Annu Rev Neurosci 21:309-345.

Frisén J, Yates PA, McLaughlin T, Friedman GC, O'Leary DD, Barbacid M (1998) Ephrin-A5 (AL-1/RAGS) is essential for proper retinal axon guidance and topographic mapping in the mammalian visual system. Neuron 20:235-243.

Frisén J, Holmberg J, Barbacid M (1999) Ephrins and their Eph receptors: multitalented directors of embryonic development. EMBO J 18:5159-5165.

Frost DO (1981) Orderly anomalous retinal projections to the medial geniculate, ventrobasal, and lateral posterior nuclei of the hamster. J Comp Neurol 203:227-256.

Godement P, Salaun J, Imbert M (1984) Prenatal and postnatal development of retinogeniculate and retinocollicular projections in the mouse. J Comp Neurol 230:552-575.

Hornberger MR, Dutting D, Ciossek T, Yamada T, Handwerker C, Lang S, Weth F, Huf J, Wessel R, Logan C, Tanaka H, Drescher U (1999) Modulation of EphA receptor function by coexpressed ephrinA ligands on retinal ganglion cell axons. Neuron 22:731-742.

Inoue T, Tanaka T, Suzuki SC, Takeichi M (1998) Cadherin-6 in the developing mouse brain: expression along restricted connection systems and synaptic localization suggest a potential role in neuronal circuitry. Dev Dyn 211:338-351.

Kalil RE, Schneider GE (1975) Abnormal synaptic connections of the optic tract in the thalamus after midbrain lesions in newborn hamsters. Brain Res 100:690-698.

Ling C, Schneider GE, Jhaveri S (1998) Target-specific morphology of retinal axon arbors in the adult hamster. Vis Neurosci 15:559-579.

Lyckman AW, Jhaveri S, Feldheim D, Frisén J, Flanagan JG, Sur M (1999) Do ephrin-A and EphA serve to compartmentalize visual and auditory structures in the developing thalamus? Soc Neurosci Abstr 24:2263.

Mackarehtschian K, Lau CK, Caras I, McConnell SK (1999) Regional differences in the developing cerebral cortex revealed by ephrin-A5 expression. Cereb Cortex 9:601-610.

Mellitzer G, Xu Q, Wilkinson DG (1999) Eph receptors and ephrins restrict cell intermingling and communication. Nature 400:77-81.

Monschau B, Kremoser C, Ohta K, Tanaka H, Kaneko T, Yamada T, Handwerker C, Hornberger MR, Loschinger J, Pasquale EB, Siever DA, Verderame MF, Muller BK, Bonhoeffer F, Drescher U (1997) Shared and distinct functions of RAGS and ELF-1 in guiding retinal axons. EMBO J 1 6:1258-1267.

Nakamoto M, Cheng HJ, Friedman GC, McLaughlin T, Hansen MJ, Yoon CH, O'Leary DD, Flanagan JG (1996) Topographically specific effects of ELF-1 on retinal axon guidance in vitro and retinal axon mapping in vivo. Cell 86:755-766.

Roe AW, Pallas SL, Hahm JO, Sur M (1990) A map of visual space induced in primary auditory cortex. Science 250:818-820.

Roe AW, Hahm J, Sur M (1991) Experimentally induced establishment of visual topography in auditory thalamus. Soc Neurosci Abstr 17:898.

Sabel BA, Schneider GE (1988) The principle of "conservation of total axonal arborizations": massive compensatory sprouting in the hamster subcortical visual system after early tectal lesions. Exp Brain Res 73:505-518

Schneider GE (1973) Early lesions of superior colliculus: factors affecting the formation of abnormal retinal projections. Brain Behav Evol 8:73-109.

Shapiro L, Colman DR (1999) The diversity of cadherins and implications for a synaptic adhesive code in the CNS. Neuron 23:427-430.

Sur M, Garraghty PE, Roe AW (1988) Experimentally induced visual projections into auditory thalamus and cortex. Science 242:1437-1441.

Suzuki SC, Inoue T, Kimura Y, Tanaka T, Takeichi M (1997) Neuronal circuits are subdivided by differential expression of type-II classic cadherins in postnatal mouse brains. Mol Cell Neurosci 9:433-447.

Upton AL, Salichon N, Lebrand C, Ravary A, Blakely R, Seif I, Gaspar $P$ (1999) Excess of serotonin (5-HT) alters the segregation of ipsilateral and contralateral retinal projections in monoamine oxidase A knock-out mice: possible role of 5-HT uptake in retinal ganglion cells during development. J Neurosci 19:7007-7024.

Vanderhaeghen P, Lu Q, Prakash N, Frisen J, Walsh CA, Frostig RD, Flanagan JG (2000) A mapping label required for normal scale of body representation in the cortex. Nat Neurosci 3:358-365.

Xu Q, Mellitzer G, Robinson V, Wilkinson DG (1999) In vivo cell sorting in complementary segmental domains mediated by Eph receptors and ephrins. Nature 399:267-271. 